\title{
Aprotinin Re-visited: Still Room for Concern
}

\author{
Laura Sommer Hansen ${ }^{1}$, Mariann Tang ${ }^{2}$, Kim Terp ${ }^{2}$ and Carl-Johan Jakobsen ${ }^{1 *}$ \\ ${ }^{1}$ Department of Anaesthesiology and Intensive Care, Aarhus University Hospital, Denmark \\ ${ }^{2}$ Department of Cardiothoracic Surgery, Aarhus University Hospital, Denmark
}

Received: 29 December, 2016; Accepted: 07 February, 2017; Published: 17 February, 2017

*Corresponding author: Carl-Johan Jakobsen, Department of Anaesthesiology and Intensive Care, Aarhus University Hospital, PalleJuul-Jensens Boulevard 99, 8200 Aarhus N, Denmark,E-mail: cjj@dadlnet.dk

\begin{abstract}
Objectives: To evaluate differences in effects of Aprotinin compared to Tranexamic acid on bleeding and severe postoperative adverse outcomes such as new thromboembolic events, dialysis, and mortality in a large cohort of high-risk patients undergoing cardiac surgery.

Methods: All adult standard on-pump cardiac surgery procedures (N=6341) from January 1, 2007 through December 31, 2014. We performed a propensity score matched analysis, aiming to match each patient receiving Aprotinin with a control receiving Tranexamic acid, ( $\mathrm{N}=513$ in each group).Primary short-term outcomes were 30day mortality, incidence of new postoperative dialysis, myocardial infarction, or stroke during the index hospitalization. Primary longterm outcomes were need for re-do coronary artery bypass grafting, coronary angiography, or percutaneous coronary intervention within six month, as well as 6-month mortality.

Results: Aprotinin patients received a significantly higher rate of red blood cell transfusion compared to patients in the control group ( $50.1 \%$ vs. $43.5 \%$; $\mathrm{p}=0.035$ ). Aprotinin was followed by a higher risk of new postoperative dialysis with a crude OR 1.78 (1.18-2.67). When adjusted for relevant confounders, the association was no longer statistically significant. Patients in the Aprotinin group had significantly increased S-creatinine levels from day one after surgery and throughout the first postoperative week. The short- and longterm mortality was comparable between groups, as were the risk of postoperative ischaemic events.
\end{abstract}

Conclusions: Aprotinin was associated with a significantly higher red blood cell transfusion rate, and an enhanced risk of impaired renal function and new dialysis compared to patients treated with Tranexamic acid, and it thus remains an unattractive alternative in high-transfusion-risk patients.

Keywords: Aprotinin; Tranexamic Acid; Cardiac Surgical Procedures; Erythrocyte Transfusion; Dialysis; Propensity Score

\section{Introduction}

Severe bleeding during and after cardiac surgery is a serious and relatively frequent complication, which may be associated with increased morbidity and mortality [1-3]. The number of patients receiving packed red blood cells (RBCs) remains high and the rate of transfusion ranges from $35 \%$ up to $66 \%$ of cardiac surgery patients [4-5]. Blood transfusion benefits patients in the acute phase, howeverentails serious risks and complications $[4,6,7]$.
Excessive bleeding may be caused by impaired haemostasis due to enhanced fibrinolysis, platelet dysfunction, haemodilution, acidosis, hypothermia, and consumption of coagulation factors in addition to the surgical trauma alone [8-9].

The anti-fibrinolytic serine protease inhibitor Aprotinin (AP) and the lysine analogue tranexamic acid (TXA) are widely used to reduce blood loss in surgery involving cardiopulmonary bypass [10-13].The mechanism of action is different as AP primarily acts by inactivating free plasmin without impact on bound plasmin, whereas TXA prevents excessive plasmin formation, and thus, prevents the binding of plasminogen to fibrin. Large metaanalyses of randomized studies have provided some evidence of a beneficial effect of fibrinolytic inhibitors [10,12]. On the contrary, other studies have suggested [14] or documented severe adverse effect after AP [15-17]. In the latter, patients were not randomized to treatment and their anti-fibrinolytic medication was part of routine clinical care.

Consequently, the use of AP was more or less abandoned in routine practice after the critical reports.

However, the increased risk of bleeding associated with complexsurgical procedures and especially fragile patientsimplies a need for drugs that have a positive effect on reducing bleeding. Thus, some surgical departments have continued the use of AP in selected patients based on individually considered potential risks and benefits.

As previous studies, including the latest publications [18-19] have not been fully conclusive and further the meta-analysis by Meybohm et al [19] all questioned the use Aprotinin in low risk patients but opened for possible treatment in high risk patients. Further, as the providers of Aprotinin are working on a general re-launch of the drug, it may be of interest whether the use of AP in a selected high-risk group may have an overall different profile, than in general routine practice.

The purpose of the present study was to evaluate differences in beneficial effects of AP compared to TXA on bleeding and severe postoperative adverse outcomes such as new thromboembolic events, dialysis, and mortality in a large cohort of high-risk patients undergoing cardiac surgery. We hypothesised that patients treated with AP would experience less bleeding but 
might face more postoperative complications than patients treated with TXA.

\section{Material and Methods}

\section{Study design}

All adult cardiac surgery patients from January 1, 2007 through December 31, 2014 from Aarhus University hospital were considered (Figure 1). Eligible procedures were in principle all standard on-pump cardiac surgery procedures, regardless of the urgency status of the surgery. However, smaller procedures like thymectomy or pericardiectomy were excluded, as were surgical procedures such as heart transplantations where all patients received AP. Additionally, patients dying within the first 48 hours were considered without impact from AP or TXA medication and were excluded together with patients with invalid ID (Civil Person Registration (CPR) number) for follow up or missing requested data, thereby leaving 6,341 patients for analysis. Patients were grouped based on treatment with AP or TXA.

\section{Patient and Outcome Characteristics}

The primary data source was The Western Denmark Heart Registry [20]. This regional registry gathers prospectively collected clinical data from all cardiothoracic procedures performed at the participating hospitals. Reporting to this webbased registry has been mandatory since its establishment in 1999, although not all data fields were compulsory in the early phase. Detailed patient-, surgery-, anaesthesia-, and intensive care-related data are reported together with in-hospital complications. Since April 2006, all data input related to this

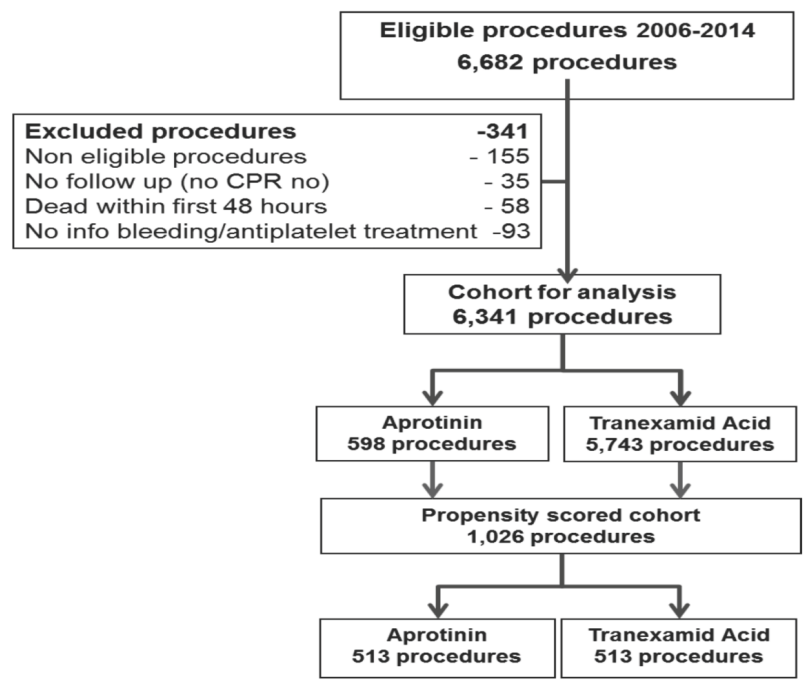

Figure 1: Patient cohort for analysis divided into two treatment groups: Aprotinin or Tranexamic acid. Non-eligible procedures were thymectomy or pericardiectomy together with patient categories always receiving Aprotinin (eg heart transplantation). No follow up due to missing CPR is patient without a valid CPR number. Missing information is primary missing data on factors possible associated with increased bleeding. analysis has been mandatory. Patient characteristics and surgical procedures are described primarily by the European System for Cardiac Operative Risk Evaluation (EuroSCORE) variables [21] and since 2013 the EuroSCORE II [22]. The Western Denmark Heart Registry obtains data on mortality from the Danish Civil Registration System [23], which keeps daily updated records on residence, migration, vital status, and date of death for all Danish citizens via the unique CPR number. The study was approved by the Danish Data Protection Agency (1-16-02-659-15). Analysing and reporting anonymous data from clinical quality databases do not require ethical approval in Denmark.

All outcome measures were in accordance with the prespecified classifications used in the Western Denmark Heart Registry. The primary direct effect parameters were the volume of postoperative bleeding, the frequency of re-exploration due to bleeding, and the transfusion of blood and blood products.

The primary short-term outcomes were the incidence of new postoperative dialysis, myocardial infarction (MI), and stroke during the index hospitalization together with the 30 day mortality (day 3 to day 30 after surgery).Definition of MI was occurrence of a new Q-wave and/or a CK-MB more than five times the upper reference level. Stroke was a combined index of both transitory ischemic attack lasting less than 24 hours and a new neurological deficit lasting more than 24 hours. We did not distinguish between haemorrhagic and is chaemic stroke. We registered postoperative acute renal failure as new need for dialysis without differentiating between the actual mode of renal replacement therapy or the precise indication for dialysis. However, changes in S-Creatinine supplemented the secondary outcome measure of renal injury. Acute Kidney Injury (AKI) was present if an increase in S-Creatinine was greater than $50 \%$ or $26.2 \mu \mathrm{mol} / \mathrm{L}$ as defined by the AKIN network [24].

The primary long-term outcomes were 6-month mortality (all deaths from day 3 within six months of surgery). Anew ischemic event defined as the need for re-do coronary artery bypass grafting (CABG), coronary angiography (CAG), or percutaneous coronary intervention (PCI) within six month of the primary operation. We did not differentiate between the specific indications for the individual interventions. However, only CAG's with the indication "suspected myocardial infarction", "unstable or stable angina pectoris", and "complication/control after CABG" were included. Coronary angiographies that could not be considered as surely ischemic e.g. "cardiac arrhythmia" or planned "Completion PCI" were excluded.

\section{Perioperative Management}

During the study period, it was standard care for patients to discontinue aspirin and oral adenosine diphosphate receptor antagonists five days prior to surgery. If indicated, patients with increased risk of ischemic events continued aspirin until the day of surgery. Vitamin K-antagonists were usually with held two to three days preoperatively. The effect was measured by blood samples the day before surgery, and antidotes were given if necessary. The surgical techniques were at the discretion of the surgical team. 
Patients received general anaesthesia with invasive haemodynamic monitoring and standardized cardiopulmonary bypass (CPB). Myocardial protection was achieved by either intermittent cold crystalloid or blood cardioplegia. The majority of patients were maintained either normothermic or mildly hypothermic. The CPB was established using a closed system consisting of tubing with a surface modifying additive coating an arterial filter with heparin coating, a hollow fibre-membrane oxygenator with a surface modified additive coating, and a venous cardiotomy reservoir. Heparin was administered to achieve an activated clotting time (ACT) greater than 400 seconds and was neutralized after CPB using protamine sulphate (Leo Pharma AS, Ballerup, Denmark). During CPB the blood flow was in general kept at $2.4 / \mathrm{L} / \mathrm{min} / \mathrm{m} 2$ and the mean arterial blood pressure 50 $70 \mathrm{~mm} \mathrm{Hg}$.

\section{Dosage of drugs and transfusions}

The standard anti-fibrinolytic treatment was TXA or in selected cases AP. The choice of antifibrinolytic agent was at the discretion of the surgeon in charge. Standardized differences before patient match indicated that aortic surgery, previous cardiac surgery, endocarditis, acute surgery, critical preoperative state and risk of long time on extra corporal circulation were factors influencing the choice of AP (Figure 2).

Patients in the TXA group received a total dosage of 4 grams at three time-points; at induction of anaesthesia $(2 \mathrm{~g})$, before CPB (1 g), and at termination of CBP (1 g). AP was administered with a loading dose of 2 million Kallikrein Inhibitor Units (KIU),2 million KIU in the heart lung machine, followed by $500.000 \mathrm{KIU}$ per hour during surgery and postoperatively, until drainage was less than $100 \mathrm{ml}$ per hour.

Residual blood from the CPB circuit is routinely re-transfused at the end of surgery. Blood products were given at the discretion of the attending anaesthesiologist or surgeon, based on local transfusion guidelines and the national recommendations for blood transfusion.

\section{Statistical Analysis}

Continuous variables were described using mean values with standard deviation or median values with interquartile ranges (IQR) and comparing analyses were done using paired samples t-test or the Wilcoxon-test/Mann-Whitney test where appropriate. Categorical variables were described as percentages and variables were compared using the $\chi 2$-test or McNemar test. A P-value less than 0.05 was considered statistically significant.

We used propensity score matching to reduce the risk of bias due to confounding and non-random assignment of transfusion therapy. The included covariate were sex, age (longitudinal), chronic obstructive pulmonary disease (COPD), peripheral artery disease, previous central nervous disease, previous surgery, s-creatinine higher than $200 \mathrm{mmol} / \mathrm{L}$, preoperative critical state, active endocarditis, preoperative cardiac factor(longitudinal; the combined score of unstable angina, left ventricular ejection fraction (3groups), recent myocardial infarction), acute surgery, aortic surgery, ventricular septum defect (VSD) surgery, all
EuroSCORE I criteria[21], operation type (CABG only, single-, double-, or triple procedure, EuroSCORE II criteria [22]), insulin dependent diabetes, continued preoperative treatment with antiplatelet drugs (APT), use of contrast plus/minus 7 days before/after surgery, and extra corporal circulation (ECC) time ( $<120$ minutes, > 120 minutes).

We performed a propensity score matched analysis, which aimed to match each patient receivingAP with a control receiving TXA with the nearest propensity score within a maximum caliper range of \pm 0.025 and without replacement. In this manner we were able to match $513(85.8 \%)$ of the 598AP patients with a control group. Covariates were adequately balanced after propensity score matching, as evidenced by a standardized difference of each covariate to values below 0.1 (Figure2).

All outcomes were described by the cumulative incidence stratified on the matched pairs. For 30-days mortality and new postoperative dialysis, we used conditional logistic regression with and without adjustment of the above mentioned covariates, to take into account the non-independency within each pair, to estimate odds ratio (OR) for the specified outcomes. We used conditional logistic regression to take into account the nonindependency within each pair when estimating odds ratio (OR) for the specified outcomes.

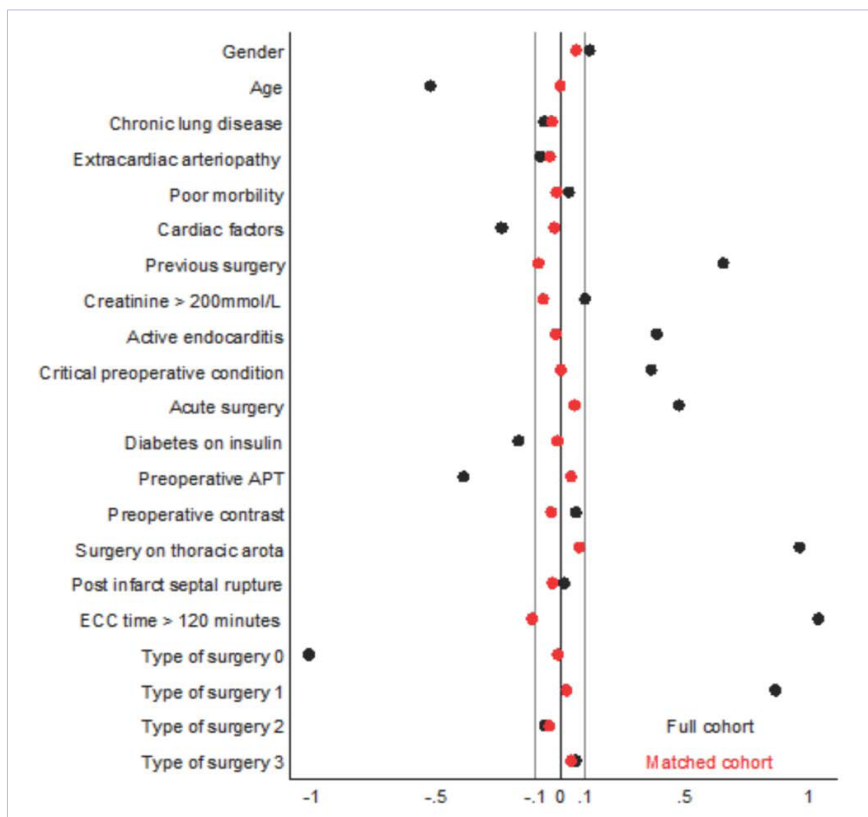

Figure 2: The standardized differences of all matching criterions before and after propensity score matching. The included covariates were; sex, age (longitudinal), chronic obstructive lung disease (COLD), extra cardiac atheropathy, preoperative central nervous system disease/poor mobility, s-creatinine $>200 \mathrm{mmol} / \mathrm{L}$, previous cardiac surgery, active endocarditis, critical preoperative state, cardiac factors (longitudinal sum of scores for unstable angina, left ventricular ejection fraction, recent myocardial infarction), acute surgery, operation type (coronary artery bypass grafting only, single-, double-, or triple procedure). All above are EuroSCORE I ${ }^{18}$ and II $^{19}$ criteria. Cardiopulmonary bypass duration (2 groups), preoperative continued antiplatelet therapy (APT) and use of contrast plus/minus 7 days before/after surgery. 
Propensity scores were performed using the statistical software package Stata ${ }^{\circledR} 12.0$ package (StataCorp LP, Texas, US).All other analyses were performed with MedCalc Statistical Software version 15.8 (MedCalc Software bvba, Ostend, Belgium; https://www.medcalc.org; 2015)

\section{Results}

During the study period, 6,682eligible consecutive patients underwent surgery at our institution (Figure 1). Out of those, 341 were excluded from the study according to the details mentioned above, leaving 6,341patients in the cohort. Of the remaining patients in the study cohort, 598 (9.4\%) received AP during and after surgery. The remaining patient receiving TXA constituted the control group. Baseline parameters and demographics of the patients before and after propensity score matching of the cohort are summarized in (Table 1).
Before the matching process, patients in the AP group were significantly different from the control group in many aspects. The patients in AP group were younger and seemed in better cardiac state (EuroSCORE cardiac factors). As negative comorbidity factors they more often had a history of previous cardiac surgery, a higher frequency of acute surgery, preoperative critical state, active endocarditis, and aortic surgery compared to TXA patients. Patients in the AP group were less likely to be exposed to antiplatelet therapy prior to surgery than the TXA patients. Overall, the EuroSCORE before match was 8 (6-11) in the AP group and 5 (3-6) in the TXA group $(\mathrm{P}<0.001$; MannWhitney). After matching, the patients in the two groups were comparable in all parameters. As a consequence of the overall higher frequency of EuroSCORE complicating factors in the AP group, the match resulted in a cohort of relative high-risk patients with a EuroSCORE of 9 (6-11) in both groups.

Table 1: Selected parameters before and after propensity score match. Dichotomous are given as numbers and percentage: $\mathrm{n}(\%) .{ }^{*} \mathrm{Mann}-\mathrm{Whitney-}$ test. All other $\chi 2$-test.

\begin{tabular}{|c|c|c|c|c|c|c|}
\hline \multirow[b]{2}{*}{ Factor } & \multicolumn{3}{|c|}{ Original cohort } & \multicolumn{3}{|c|}{ After propensity match } \\
\hline & Aprotinin & Control & p-value & Aprotinin & Control & p-value \\
\hline No of patients & $\mathrm{n}=598$ & $n=5743$ & & $n=513$ & $n=513$ & \\
\hline Sex, male & $397(66)$ & $4112(72)$ & 0,007 & $177(35)$ & $161(31)$ & 0.288 \\
\hline Age, mean (sd) & $58.8(17.0)$ & $65.8(12.7)$ & $0.000^{*}$ & $59.1(17.1)$ & $59.1(16.2)$ & 0.706 \\
\hline Cronic lung disease & $63(11)$ & $706(12)$ & 0.215 & $52(10)$ & $57(11)$ & 0.612 \\
\hline Extracardiacarteriopathy & $35(6)$ & $442(8)$ & 1.105 & $29(6)$ & $34(7)$ & 0.516 \\
\hline Poor mobility & $46(8)$ & $390(7)$ & 0.398 & $42(8)$ & $44(9)$ & 0.822 \\
\hline Cardiac factor-score, mean (sd) & $0.59(1.2)$ & $0.90(1.4)$ & $0.000^{*}$ & $0.60(1.2)$ & $0.63(1.2)$ & 0.643 \\
\hline Previous surgery & $175(29)$ & $316(6)$ & 0.000 & $139(27)$ & $159(31)$ & 0.169 \\
\hline Creatinine $>200 \mathrm{mmol} / \mathrm{l}$ & $30(5)$ & $176(3)$ & 0.01 & $26(5)$ & $34(7)$ & 0.287 \\
\hline Active endocarditis & $86(14)$ & $200(3)$ & 0.000 & $77(15)$ & $80(16)$ & 0.795 \\
\hline Critical preoperative condition & $108(18)$ & $358(6)$ & 0.000 & $81(16)$ & $80(16)$ & 0.932 \\
\hline Acute surgery & $144(24)$ & $413(7)$ & 0.000 & $112(22)$ & $100(19)$ & 0.355 \\
\hline Diabetes on insulin & $14(2)$ & $323(6)$ & 0.001 & $13(3)$ & $14(3)$ & 0.845 \\
\hline Preoperative APT & $42(7)$ & $1143(20)$ & 0.000 & $39(8)$ & $33(6)$ & 0.463 \\
\hline Preoperative contrast & $111(19)$ & $926(16)$ & 0.125 & $95(19)$ & $102(20)$ & 0.579 \\
\hline Surgery on thoracic aorta & $234(40)$ & $204(4)$ & 0.000 & $158(31)$ & $140(27)$ & 0.216 \\
\hline Post infarct septal rupture & $2(0.3)$ & $14(0.2)$ & 0.674 & $2(0.4)$ & $3(0.6)$ & 0.654 \\
\hline ECC time $>120 \mathrm{~min}$ & $426(71)$ & $1436(25)$ & 0.000 & $343(67)$ & $369(72)$ & 0.078 \\
\hline \multicolumn{7}{|l|}{ Type of surgery } \\
\hline Single CABG & $25(4)$ & $2423(42)$ & \multirow{4}{*}{0.000} & $25(5)$ & $26(5)$ & \multirow{4}{*}{0.820} \\
\hline Single non-CABG & $448(75)$ & $2034(35)$ & & $368(72)$ & $362(71)$ & \\
\hline Two procedures & $105(18)$ & $1153(20)$ & & $101(20)$ & $110(21)$ & \\
\hline Three procedures & $20(3)$ & $133(2)$ & & $19(4)$ & $15(3)$ & \\
\hline High Euro-SCORE & $223(37)$ & $841(15)$ & 0.000 & $185(36)$ & $181(35)$ & 0.794 \\
\hline Perioperative Hydroxy-ethyl starch & $337(56)$ & $3442(65)$ & 0.002 & 300 (58) & 313 (61) & 0.408 \\
\hline
\end{tabular}


The effect parameters are listed in table 2. The patients in AP group were significantly more likely to receive RBC transfusion compared to the patients in the TXA group (50.1\% vs. $43.5 \%$; $\mathrm{p}=0.035$ ). The same tendency, although non-significant, was seen for plasma transfusions. No difference was seen in the transfused volume between the groups. Patients receiving AP showed indications of lower postoperative drainage, less frequent re-exploration due to bleeding and were less often treated with fibrinogen concentrate or recombinant factor VIIa (Novoseven $\AA$ ) (Table 2). However, none of the findings were statistically significant.

Outcomes were analyzed using conditional regression analysis (Table 3 ). The crude regression analysis demonstrated that use of AP had a higher risk of new postoperative dialysis with OR 1.78 (1.18-2.67). However, when the data was adjusted for perioperative use of inotropes, vasoconstrictors, fibrinogen concentrate and Novo Seven $\AA$, as well as transfusion of allogeneic blood products, this impact was no longer present (OR $1.26(0.71-2.23)$ ). As illustrated in (Figure 3), patients in the AP group had increased S-Creatinine levels from day one after surgery and throughout the first postoperative week $(p<0.0001$ $-p=0.0092$ ). The fraction of patients with AKI was significantly higher in patients receiving AP than in patients treated with TXA (Table 4).

None of the individual risk factor shad independent impact on 30-day mortality (Table 5). Perioperative vasoconstrictors, and transfusion of blood and blood products had individual impact on new postoperative dialysis and 6 month mortality, while the only factor with independent impact on late ischaemic events was perioperative use of inotropes.

\section{Discussion}

Despite several critical reports concerning the use of AP in cardiac surgery $[14,25]$, the prevailing opinion is that AP is superior to TXA and therefore may be used in high-transfusionrisk patients [26]. Nevertheless, the present propensity-matched case-control study demonstrates an association between the administration of AP and a greater need for perioperative RBC, contrasting the alleged effect of the drug.

The overall transfusion rate in the study was high, confirming that the patients were sampled from a high-transfusion-risk population. By modelling the exposure rather than the outcome, propensity scores efficiently allow for simultaneous control for a large number of potentially confounding factors [27], and application of the model made patients in the two groups comparable in all parameters after matching.

Additionally, the AP patients showed a trend towards a higher demand for plasma transfusion. The increased utilization of RBCs and plasma is disturbing, since the transfusion of allogeneic blood products during cardiac surgery is known to increase the concentration of pro-inflammatory mediators that are associated with development of multiple organ dysfunction syndrome and hospital mortality [28]. Furthermore, the transfusion of RBC has been shown to be the factor most consistently associated with an increased risk of postoperative morbidity and mortality $[29,30]$.

AP has previously been demonstrated to be associated with postoperative renal dysfunction in a comparable patient population [14]. Examining S-Creatinine levels in the cohort, we found a significant increase in AKI amongst patients treated with AP. The difference was sustained with time from surgery, discarding a mere transient effect on renal function. However, the higher S-Creatinine and the following higher frequency of AKI had no impact on the later findings like new dialysis and mortality.

Table 2: Effect drainage and transfusion of Aprotinin compared to tranexamic acid in propensity score matched patients. \#) Wilcoxontest !) McNemar test *) Mann-Whitney test.

\begin{tabular}{|c|c|c|c|c|c|c|c|c|c|c|}
\hline \multirow[b]{2}{*}{ Group } & \multirow[b]{2}{*}{ Drainage } & \multirow{2}{*}{$\begin{array}{l}\text { Re-do } \\
\text { surgery }\end{array}$} & \multicolumn{2}{|c|}{ Administration of } & \multicolumn{2}{|c|}{ Red Blood Cells } & \multicolumn{2}{|l|}{ Plasma } & \multicolumn{2}{|l|}{ Platelets } \\
\hline & & & Fibrinogen & $\begin{array}{l}\text { Novo } \\
\text { Seven }\end{array}$ & No & Volume & No & Volume & No & Volume \\
\hline Aprotinin & $489(280-976)$ & $41(8.0)$ & $50(9.7)$ & $18(3.5)$ & $257(50.1)$ & $1200(600-2700)$ & $257(50.1)$ & $1200(600-2400)$ & $226(44.0)$ & $600(300-900)$ \\
\hline $\begin{array}{l}\text { Tranexamic } \\
\text { Acid }\end{array}$ & $513(310-1145)$ & $48(9.4)$ & $53(10.3)$ & $25(4.9)$ & $223(43.5)$ & $1200(600-2700)$ & $227(44.2)$ & $1200(600-2400)$ & $204(39.8)$ & $600(300-1200)$ \\
\hline
\end{tabular}

Table 3: Conditional regression analysis of propensity score matched data. Parameters in adjusted analysis are Aprotinin together with perioperative use of vasoconstrictors, inotropes, fibrinogen, NovoSeven ${ }$, and transfusion of blood or blood products.

\begin{tabular}{|c|c|c|c|}
\hline Outcome parameter & \multicolumn{2}{|c|}{ Cumulative index } & \multicolumn{2}{c|}{ Conditional regression analysis } & Crude 0R (95 \% CI) & Adjusted OR (95 \% CI) \\
\hline 30-day mortality & Aprotinin & Tranexamid acid & $0.96(0.37-2.47)$ \\
\hline New postoperative dialysis & $20(3.9)$ & $21(4.1)$ & $1.78(1.18-2.67)$ \\
\hline Postoperative stroke & $10(13.6)$ & $42(8.2)$ & $0.50-1.81)$ \\
\hline Postoperative MI & $13(2.5)$ & $22(4.3)$ & $1.26(0.71-2.23)$ \\
\hline 6mth Ischaemic event & $30(5.8)$ & $13(2.5)$ & $0.93(0.39-2.19)$ \\
\hline 6mth mortality & $42(8.2)$ & $45(8.8)$ & $0.98(0.43-2.22)$ \\
\hline
\end{tabular}


The crude conditional regression analyses demonstrated a statistically significant increase in the need for postoperative dialysis amongst patients treated with AP. Adjusting the data for perioperative use of inotropes, vasoconstrictors, fibrinogen concentrate and Novo Seven $\AA$, as well as transfusion of any blood product, the impact was only trending. However, transfusion of allogeneic blood products was in itself significantly associated with new dialysis, presumably diminishing the isolated impact caused by administration of the drug.

We found no differences in short- and long-term mortality between groups, nor did we detect any difference in the number of postoperative ischaemic events. TXA has demonstrated to be a safe antifibrinolytic treatment, despite recent reports of increased incidence of seizures [31]. In this light, the authors believe that the greater need for allogeneic blood products as well as the association to impairment of renal function in patients treated with AP is a cause for concern. The neutral frequency of postoperative complications in AP group compared to TXA group is better than our previous report of general use [16], and in alignment with the most resent meta-analysis [19] of high-risk patients.

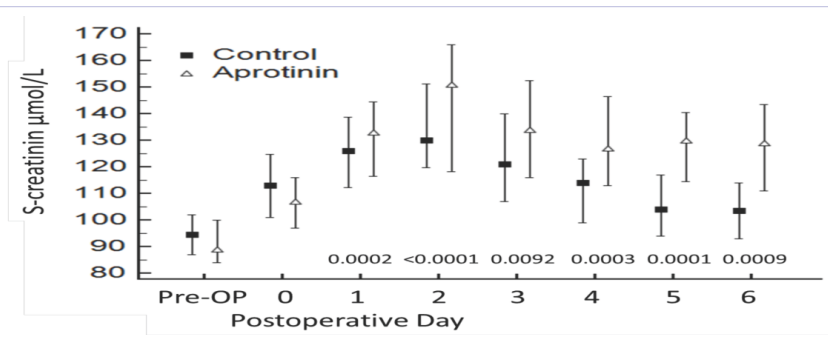

Figure 3: S-creatinine levels preoperatively and the first 6 postoperative days divided on treatment groups. The levels were statistically significant different all days from day 1 and forward (Mann-Whiney test).

Table 4: Fraction of patients with acute kidney injury (increase in S-Creatinine greater than $50 \%$ or $26.2 \mu \mathrm{mol} / \mathrm{L}$ ) based on treatment group and evaluated 3, 6 and 9 days after surgery. Statistics are $\chi 2$-test.

\begin{tabular}{|c|c|c|c|}
\hline \multirow{2}{*}{ Group } & \multicolumn{3}{|c|}{ Number of patients with AKI } \\
\cline { 2 - 4 } & $\mathbf{3}$ days & $\mathbf{6}$ days & 9 days \\
\hline Aprotinin & $43,80 \%$ & $47,90 \%$ & $51,20 \%$ \\
\hline Tranexamid Acid & $35,20 \%$ & $36,50 \%$ & $37,80 \%$ \\
\hline & $\mathrm{P}=0.0074$ & $\mathrm{P}=0.0004$ & $\mathrm{P}<0.0001$ \\
\hline
\end{tabular}

\section{Limitations of the study}

Some limitations must be addressed. Being an observational study, the allocation of patients was not randomly determined. Although propensity score matching aims to achieve a quasirandomized design, the method is highly dependent on the choice of variables. However, by stratifying and conditioning, we took the non-independency within each matched pair into account [32]. Potentially, we could have discarded variables reflecting between-groups differences, which would bias our results. Furthermore, as the transfusion practice in the present study was not controlled but directed by clinical guidelines, the patients allocated to AP treatment could be more exposed to a so-called prophylactic transfusion practice. This unwanted side effect is underlined by the fact that numbers transfused was higher in AP patients although the postoperative bleeding was the same as TXA patients. Further, the mere decision to treat a patient with AP could reinforce the perception of the patient as being in high risk of perioperative bleeding, thereby tipping the scales in favour of transfusion in borderline cases. This may especially apply to plasma and platelet transfusion as such blood products are sometimes given to prevent bleeding in high-risk individuals. $\mathrm{RBC}$ is given in case of ongoing or uncontrollable bleeding, and it is additionally guided by haemoglobin measures, assumedly downscaling the risk bias associated with treatment allocation.

A well-known risk in propensity scores match is the risk of excluding significant numbers or relevant special cases. In this study 513 of $598(85.8 \%)$ AP patients were included which is relatively high fraction, thus, diminishing the above-mentioned problem.

\section{Conclusion}

Patients treated with AP had a significantly higher RBC transfusion rate compared to patients treated with TXA, illustrating that the effect of APon haemostasis is at best comparable to TXA. The short- and long-term mortality was comparable between groups, as were the risk of postoperative ischaemic events. However, the trend towards affected renal function in AP patients is still cause for concern.

Conclusively, AP remains an unattractive alternative to TXA in high-transfusion-risk patients.

Funding sources and disclosures

This study was founded by Aarhus University Hospital. The authors have no competing interests to declare.

Table 5: Adjusted conditional regression analysis of propensity score matched data showing individual impact of parameters used in adjustment.

\begin{tabular}{|c|c|c|c|c|}
\hline Outcome factor & 30-day mortality & New dialysis & 6mth mortality & 6mth ischaemic event \\
\hline Perioperative Aprotinin & $0.96(0.37-2.47)$ & $1.26(0.71-2.23)$ & $0.81(0.46-1.45)$ \\
\hline Perioperative constrictors & $7.02(0.74-67.0)$ & $6.31(2.08-19.2)$ & $3.90(1.24-12.3)$ \\
\hline Perioperative inotropes & $1.96(0.07-56.9)$ & $0.93(0.18-4.68)$ & $1.36(0.28-6.62)$ \\
\hline Perioperative fibrinogen & $1.92(0.10-35.8)$ & $1.23(0.38-4.01)$ & $1.67(0.33-8.59)$ & $0.94(0.39-2.29)$ \\
\hline Perioperative Novo Seven & $0.26(0.01-4.84)$ & $0.73(0.15-3.63)$ & $0.51(0.08-3.26)$ & $4.71(0.41-54.6)$ \\
\hline Blood and blood products & $4.51(0.86-23.6)$ & $8.14(2.33-28.5)$ & $3.37(1.13-10.0)$ \\
\hline
\end{tabular}




\section{References}

1. Moulton MJ, Creswell LL, Mackey ME, Cox JL, Rosenbloom M Reexploration for bleeding is a risk factor for adverse outcomes after cardiac operations. J Thorac Cardiovasc Surg. 1996;111(5):1037-46.

2. Spiess BD. Transfusion of blood products affects outcome in cardiac surgery. Semin Cardiothorac Vasc Anesth. 2004;8(4):267-81.

3. Dacey LJ, Munoz JJ, Baribeau YR, Johnson ER, Lahey SJ, Leavitt BJ et al. Reexploration for hemorrhage following coronary artery bypass grafting: incidence and risk factors. Northern New England Cardiovascular Disease Study Group. Arch Surg. 1998;133(4):442-7.

4. Jakobsen CJ, Ryhammer PK, Tang M, Andreasen JJ, Mortensen PE. Transfusion of blood during cardiac surgery is associated with higher long-term mortality in low-risk patients. Eur J Cardiothorac Surg. 2012;42(1):114-20.

5. Brown JR, Birkmeyer NJ, O'Connor GT. Meta-analysis comparing the effectiveness and adverse outcomes of antifibrinolytic agents in cardiac surgery. Circulation. 2007;115(22):2801-13.

6. Chelemer SB, Prato BS, Cox PM, Jr., O’Connor GT, Morton JR. Association of bacterial infection and red blood cell transfusion after coronary artery bypass surgery. Ann Thorac Surg. 2002;73(1):138-42.

7. Speiss BD. Transfusion and outcome in heart surgery. Ann Thorac Surg. 2002;74:986-7.

8. Despotis GJ, Avidan MS, Hogue CW, Jr. Mechanisms and attenuation of hemostatic activation during extracorporeal circulation. Ann Thorac Surg. 2001;72(5):S1821-31.

9. Paparella D, Brister SJ, Buchanan MR. Coagulation disorders of cardiopulmonary bypass: a review. Intensive Care Med. 2004;30(10):1873-81.

10. Levi M, Cromheecke ME, de Jonge E, Prins MH, de Mol BJ, Briet E et al Pharmacological strategies to decrease excessive blood loss in cardiac surgery: a meta-analysis of clinically relevant endpoints. Lancet. 1999;354(9194):1940-7.

11. Nuttall GA, Oliver WC, Ereth MH, Santrach PJ, Bryant SC, Orszulak TA et al. Comparison of blood-conservation strategies in cardiac surgery patients at high risk for bleeding. Anesthesiology. 2000;92(3):674-82

12. Sedrakyan A, Treasure T, Elefteriades JA. Effect of aprotinin on clinical outcomes in coronary artery bypass graft surgery: a systematic review and meta-analysis of randomized clinical trials. J Thorac Cardiovasc Surg. 2004;128(3):442-8.

13. Royston D. The current place of aprotinin in the management of bleeding. Anaesthesia. 2015;70 Suppl 1:46-9, e17.

14. Karkouti K, Beattie WS, Dattilo KM, McCluskey SA, Ghannam M, Hamdy A et al. A propensity score case-control comparison of aprotinin and tranexamic acid in high-transfusion-risk cardiac surgery. Transfusion. 2006;46(3):327-38.

15. Mangano DT, Tudor IC, Dietzel C. Multicenter Study of Perioperative Ischemia Research $\mathrm{G}$, Ischemia $\mathrm{R}$, Education $\mathrm{F}$. The risk associated with aprotinin in cardiac surgery. N Engl J Med. 2006;354(4):353-65.

16. Jakobsen CJ, Sondergaard F, Hjortdal VE, Johnsen SP. Use of aprotinin in cardiac surgery: effectiveness and safety in a population-based study. Eur J Cardiothorac Surg. 2009;36(5):863-8.

17. Mangano DT, Miao Y, Vuylsteke A, Tudor IC, Juneja R, Filipescu D et al. Mortality associated with aprotinin during 5 years following coronary artery bypass graft surgery. JAMA. 2007;297(5):471-9.
18. Hutton B, Joseph L, Fergusson D, Mazer CD, Shapiro S, Tinmouth A. Risks of harms using antifibrinolytics in cardiac surgery: systematic review and network meta-analysis of randomised and observational studies. BMJ. 2012;345:e5798.

19. Meybohm P, Herrmann E, Nierhoff J, Zacharowski K. Aprotinin may increase mortality in low and intermediate risk but not in high risk cardiac surgical patients compared to tranexamic acid and epsilonaminocaproic acid -- a meta-analysis of randomised and observational trials of over 30.000 patients. PLoS One. 2013;8(3):e58009.

20.Schmidt M, Maeng M, Jakobsen CJ, Madsen M, Thuesen L, Nielsen PH et al. Existing data sources for clinical epidemiology: The Western Denmark Heart Registry. Clin Epidemiol. 2010;2:137-44.

21. Roques F, Nashef SA, Michel P, Gauducheau E, de Vincentiis C, Baudet $\mathrm{E}$ et al. Risk factors and outcome in European cardiac surgery: analysis of the EuroSCORE multinational database of 19030 patients. Eur J Cardiothorac Surg. 1999;15(6):816-22.

22. Nashef SA, Roques F, Sharples LD, Nilsson J, Smith C, Goldstone AR et al. EuroSCORE II. Eur J Cardiothorac Surg. 2012;41(4):734-44.

23. Pedersen CB. The Danish Civil Registration System. Scand J Public Health. 2011;39(7 Suppl):22-5

24. Mehta RL, Kellum JA, Shah SV, Molitoris BA, Ronco C, Warnock DG et al. Acute Kidney Injury Network: report of an initiative to improve outcomes in acute kidney injury. Crit Care. 2007;11(2):R31.

25. Henry DA, Carless PA, Moxey AJ, O’Connell D, Stokes BJ, Fergusson DA et al. Anti-fibrinolytic use for minimising perioperative allogeneic blood transfusion. Cochrane Database Syst Rev. 2011;(1):CD001886

26. Ferraris VA, Saha SP, Oestreich JH, Song HK, Rosengart T, Reece TB et al. 2012 update to the Society of Thoracic Surgeons guideline on use of antiplatelet drugs in patients having cardiac and noncardiac operations. Ann Thorac Surg. 2012;94(5):1761-81.

27. Sturmer T, Joshi M, Glynn RJ, Avorn J, Rothman KJ, Schneeweiss S. A review of the application of propensity score methods yielded increasing use, advantages in specific settings, but not substantially different estimates compared with conventional multivariable methods. J Clin Epidemiol. 2006;59(5):437-47.

28. Bilgin YM, van de Watering LM, Versteegh MI, van Oers MH, Brand A Effects of allogeneic leukocytes in blood transfusions during cardiac surgery on inflammatory mediators and postoperative complications. Crit Care Med. 2010;38(2):546-52

29. Murphy GI, Reeves BC, Rogers CA, Rizvi SI, Culliford L, Angelini GD. Increased mortality, postoperative morbidity, and cost after red blood cell transfusion in patients having cardiac surgery. Circulation. 2007;116(22):2544-52

30. Koch CG, Li L, Duncan AI, Mihaljevic T, Cosgrove DM, Loop FD et al. Morbidity and mortality risk associated with red blood cell and bloodcomponent transfusion in isolated coronary artery bypass grafting. Crit Care Med. 2006;34(6):1608-16.

31. Murkin JM, Falter F, Granton J, Young B, Burt C, Chu M. High-dose tranexamic Acid is associated with nonischemic clinical seizures in cardiac surgical patients. Anesth Analg. 2010;110(2):350-3.

32. Austin PC. Propensity-Score Matching in the Cardiovascular Surgery Literature from 2004 to 2006: A Systematic Review and Suggestions for Improvement. J Thorac Cardiovasc Surg. 2007;134(5):1128-1135 